\title{
Radiation background simulation and verification at the LHC: Examples from the ATLAS experiment and its upgrades
}

\author{
Ian Dawson* \\ On behalf of the ATLAS Inner Detector \\ University of Sheffield \\ E-mail: Ian.Dawsonecern.ch
}

The high collision rates at the new energy and luminosity regime of the Large Hadron Collider (LHC) gives rise to unprecedented radiation environments, especially in the inner regions of the experiments. High fidelity particle transport codes such as FLUKA and GEANT4 are necessary for simulating the complex radiation environments in detail. The results are used for predicting detector system behaviour and performance over the lifetime of the project. In this paper the Monte Carlo tools used to simulate the radiation backgrounds are discussed and examples of the predictions in the ATLAS inner detector regions are shown. Also presented are the results of verification studies in which the fluence and dose predictions are compared with measurements using recent LHC data. It is shown that the level of agreement has exceeded expectations, with differences typically less than $30 \%$, and always smaller than a factor of two. Finally, the radiation background situation for the ATLAS inner tracker at the future high luminosity upgrade of the LHC (HL-LHC) is discussed, as well as a simple parameterisation of silicon damage fluences to cover all collision energies.

The 21st International Workshop on Vertex Detectors

16-21 September 2012

Jeju, Korea

\footnotetext{
* Speaker.
} 


\section{Introduction}

The high collision rates at the new energy and luminosity regime of the LHC gives rise to extreme radiation environments, especially in the inner regions of the experiments. Deleterious effects of radiation on the experiments include: damage to detectors and electronics; fake backgrounds in the selection and reconstruction of interesting physics events; single event upsets causing disruption to data; radioactivation of components making access for maintenance difficult. High fidelity codes such as FLUKA [1] and GEANT4 [2] are necessary for simulating the complex radiation background environments in detail. The results are then used for predicting detector system behaviour and performance over the lifetime of the project. In this paper the following topics are covered: First the Monte Carlo tools used to simulate the radiation backgrounds are discussed, which include the particle transport codes FLUKA and GEANT4, as well as the collision event generators PHOJET/DPMJET [3] and PYTHIA [4]. Examples from the ATLAS experiment [5] will be shown, focusing on the inner detector regions. The second part of the paper will deal with the verification of these simulations, in which the fluence and dose predictions are compared with measurements. Finally, examples of radiation background studies for future upgrades at the HL-LHC are presented.

\section{Simulations at the $\mathrm{LHC}$}

Radiation environments at the LHC and its upgrades are complex, comprising a full spectrum of particles (pion, proton, neutron, photon, electron, muon etc.), with energies ranging from $\mathrm{TeV}$ down to thermal in the case of neutrons. The most reliable method for studying radiation backgrounds at high energy hadron collider experiments is through Monte Carlo (MC) simulation.

The simulation starts with the generation of inelastic proton-proton events. Two main MC event generators are used by the LHC experiments to simulate the collisions: PYTHIA and PHOJET1.12/DPMJET-III ${ }^{1}$. These two MC's have well developed models for describing both the soft and hard QCD components in proton-proton interactions. Inclusive inelastic measurements taken at the LHC have allowed tests, tunes and validation of the event generators. Cross-section measurements of the inelastic collisions have also been made and compared with the MC predictions [6]. The particles from the event generators are fed into the FLUKA or GEANT4 simulation. Accurate modelling of the detector geometry and material is crucial, including the regions outside the inner detector material (for example: calorimeters, shieldings, beamline equipment).

From these simulations particle fluences and doses for studying effects on detectors and personnel can be obtained. For example, charged particle fluences are useful for estimating detector occupancies, whereas hadron fluence with energy greater than $20 \mathrm{MeV}$ allows the study of single event upsets. Ionising dose is the quantity of interest for understanding surface damage effects in electronics. Thermal neutrons are important for investigating radioactivation issues. Perhaps the most important quantity of interest in the inner detector regions is the $1 \mathrm{MeV}$ neutron equivalent fluence $\left(\Phi_{1 \mathrm{MeV}}\right)$, which is needed for determining bulk damage to silicon detectors. These are obtained by weighting the particle spectra with energy dependent damage curves. Previous inner

\footnotetext{
${ }^{1}$ DPMJET-III utilises PHOJET-1.12 for hadron-hadron collisions.
} 
detector studies by ATLAS have shown that the radiation backgrounds are dominated close to interaction point by particles coming directly from the proton-proton collisions, but at larger radii by particles originating from high energy hadron and electromagnetic cascades in the calorimeters. A full and detailed description of all the radiation background studies performed for ATLAS operation at the LHC can be found in [7].

\subsection{Comparison of FLUKA with GEANT4}

The FLUKA code is well established in studies of hadronic and electromagnetic cascades in matter, employing advanced physics models for treating interactions for all particles in the $\mathrm{keV} \rightarrow \mathrm{TeV}$ range, except for neutrons which are transported down to thermal energies. GEANT4 is similar, but a more recent development, and is the baseline code of choice at the LHC experiments for simulating hard scatter physics processes. There is motivation to use GEANT4 also for future LHC upgrade simulations so comparisons with FLUKA are useful.

Shown in Figure 1 are examples of fluence energy spectra for photons and neutrons in the inner regions of the ATLAS detector. In both the FLUKA and GEANT4 simulations the same PYTHIA6 generated events are used. Pions dominate particle production in the inelastic proton-proton interactions. The high energy photons seen in the left plot of Figure 1 are from the decay of neutral pions - the excellent agreement is expected as most of these pions come directly from the interaction point. This is a also useful cross check that the event generator set-up is the same in both simulations. Below $20 \mathrm{MeV}$ the photon spectrum is dominated by gammas from neutron capture and differences in the FLUKA and GEANT4 predictions can be seen. This is probably due to the detector material descriptions which, although similar, are non-identical, and low energy neutron transport and capture is very sensitive to material composition. The discrepancies observed in the high energy neutrons, however, are more likely due to differences in the complex physics models implemented in FLUKA and GEANT4.

Photon spectrum

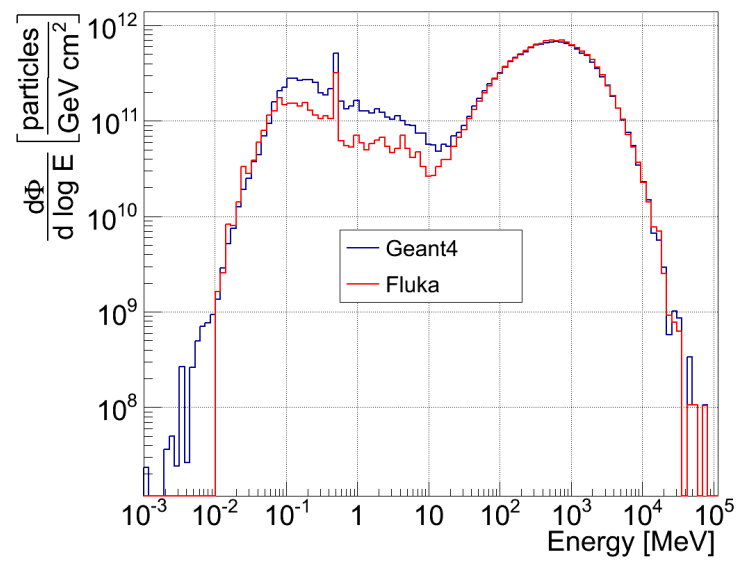

Neutron spectrum

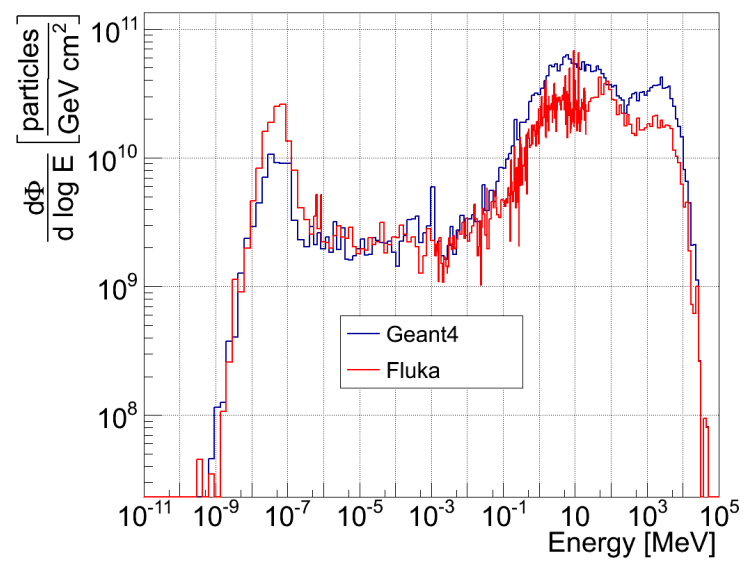

Figure 1: Photon and neutron energy spectra in the Pixel detector regions. The lower number of low energy (thermal) neutrons in the right plot indicates higher neutron capture reactions in the GEANT4 simulations, leading to the increased number of the low energy photons, as seen in the left plot. 
When convolving the energy spectra with the silicon displacement damage functions to produce $1 \mathrm{MeV}$ neutron equivalent fluences, it was found that differences between the FLUKA and GEANT4 simulations are typically less than $30 \%$. However, it is seen from the energy spectra that differences of a factor two can be observed at certain energies, and this should be kept in mind when studying the impact of radiation backgrounds on detector systems.

\section{Comparison of measurement with simulation}

Comparison of radiation background measurements with the simulated predictions is important for three reasons. First, the design of the detector systems has relied on MC simulation for predicting performance over the lifetime of the LHC, so it is crucial that assumptions are checked. Second, there is strong motivation to improve the accuracy of the simulations for future upgrade studies, so that design safety factors can be reduced. Finally, the comparisons will provide valuable input data to the developers of the simulation codes.

In this section we describe some of the measurements made by the ATLAS inner detector in 2011. The detector layout is shown in Figure 2. Of particular interest for these studies are the leakage current increases in the Pixel and silicon strip (SCT) detectors. These allow the determination of $\Phi_{1 \mathrm{MeV}}$ from the relation $\Delta I(t, T)=\alpha(t, T) \cdot V \cdot \Delta \Phi_{1 \mathrm{MeV}}$, where $\Delta I$ is the measured increase in current due to $\Delta \Phi_{1 \mathrm{MeV}} ; \alpha(t, T)$ is the current damage factor which depends on the temperature $T$ and annealing time $t$, and is known from irradiation studies [8,9]. The parameter $V$ is the detector volume. Further information on the ATLAS Pixel and SCT measurements is given in [10].

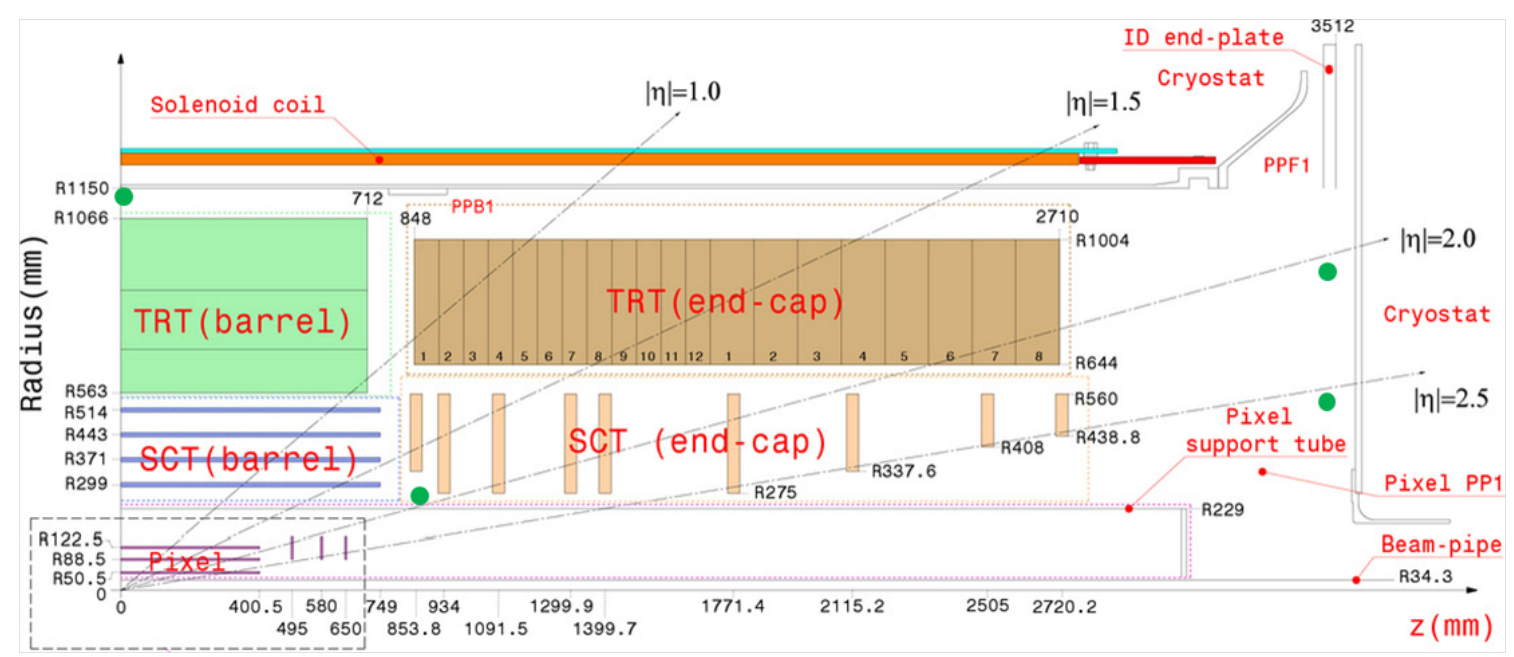

Figure 2: Schematic diagram showing a quarter-section of the ATLAS inner detector and the locations of the Pixel and SCT detectors [5]. Indicated by the green dots are the locations of the RadMons.

Shown in Figure 3 are the ratios of measured $\Phi_{1 \mathrm{MeV}}$ to simulated in the SCT system. The baseline code for simulating ATLAS inner detector radiation backgrounds is FLUKA. Excellent agreement is seen in the SCT barrel regions. Good agreement is seen over most of the SCT regions, with differences typically less than $25 \%$. The larger discrepancies seen for the inner modules of SCT end-caps, up to a factor two, are not understood and are under investigation. 
It should be noted that the simulations in Figure 3 were performed using the PHOJET event generator. Recent studies with tuned PYTHIA8 predict $\sim 10 \%$ higher fluences in the end-caps, suggesting some of the discrepancy is due to the modelling of the proton-proton interaction.

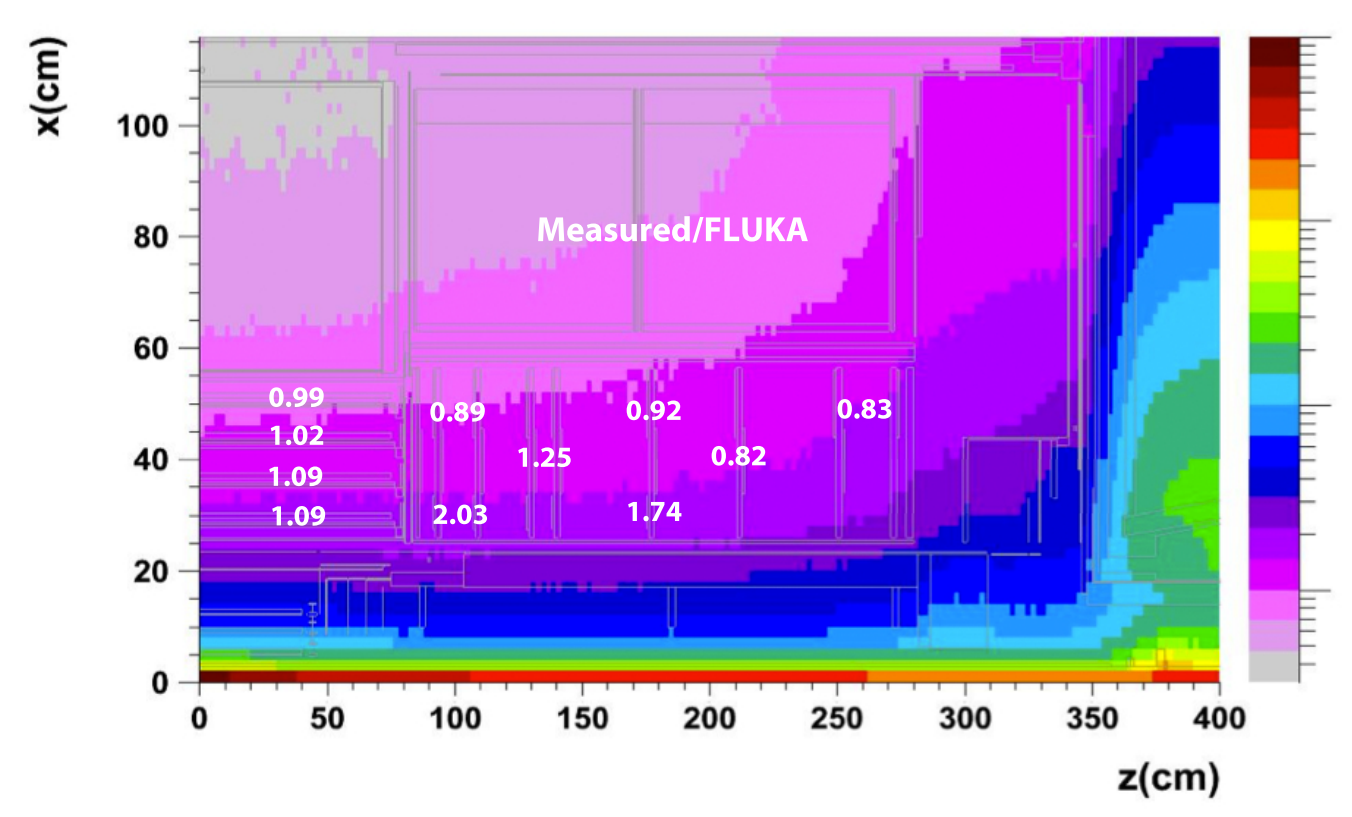

$10^{11}$

Figure 3: Quarter slice through the ATLAS inner detector region of the geometry constructed in the FLUKA simulations. The colour contours show the $1 \mathrm{MeV}$ neutron equivalent fluences corresponding to $\sqrt{s}=7 \mathrm{TeV}$, and an integrated luminosity of $1 \mathrm{pb}^{-1}$. The numbers indicate the measured to simulated fluence ratios in the SCT barrel and end-caps. The event generator used in these simulations was PHOJET-1.12.

For the Pixel barrel detectors the predictions require scaling up by $15-25 \%$ to agree with the measurements [10], which is similar to the differences seen in the majority of the SCT measurements. It is worth noting that the $1 \mathrm{MeV}$ neutron equivalent damage fluences in the SCT regions are dominated by neutrons originating from hadronic interactions in the calorimeters, whereas in the Pixels the fluences are dominated by the proton-proton collision products. Hence the Pixel and SCT measurements provide complementary information about the radiation backgrounds in the inner detector.

In addition to the silicon detector leakage current measurements, dedicated radiation monitoring devices (RadMons) were installed in locations in the ATLAS inner detector, see Figure 2. The RadMons comprise p-i-n diodes calibrated to measure $\Phi_{1 \mathrm{MeV}}$, and RadFETs used for measuring Ionising Dose, which is important for understanding damage to electronics. DMILL bipolar transistors are also included, calibrated for measuring thermal neutron fluence. Excellent agreement is observed between measurements and simulated predictions, with differences typically less than $30 \%$. For further details see [11]. 


\section{Simulation and predictions at the HL-LHC and beyond.}

At the HL-LHC a factor ten increase in integrated luminosity for physics is planned, leading to similar increases in radiation backgrounds. The good agreement between simulation and measurement described in Section 3 gives increased confidence for future upgrade studies. Shown in Figure 4 are the $1 \mathrm{MeV}$ neutron equivalent fluence predictions from FLUKA corresponding to the latest ATLAS upgrade inner tracker design [12], normalised to $3000 \mathrm{fb}^{-1}$. All the ATLAS detector material in the simulation has to be accurately modelled, including services, calorimeters, shieldings etc. The event generator used in these studies is PYTHIA8, to take advantage of the latest 7-8 TeV ATLAS minimum bias tunings. Close to the interaction point the backgrounds are dominated by particles coming directly from the proton-proton collisions, but at larger radii neutrons originating from high energy hadron cascades in the calorimeter material dominate.

In the ATLAS inner tracker systems the most extreme damage fluences are seen in the barrel layer closest to the interaction point at $r=3.9 \mathrm{~cm}$, with a predicted $\Phi_{1 \mathrm{MeV}}$ value of $1.4 \times 10^{16}$ $\mathrm{cm}^{-2}$. The outer two layers of the pixel barrel and the pixel disks will use existing silicon planar technology and a maximum fluence of $1.7 \times 10^{15} \mathrm{~cm}^{-2}$ is predicted (in the third barrel layer at $r=15.5 \mathrm{~cm})$. The maximum $\Phi_{1 \mathrm{MeV}}$ fluence in the silicon strip system is predicted to be in the end-caps $(z=300 \mathrm{~cm})$ with a value $8.1 \times 10^{14} \mathrm{~cm}^{-2}$, and in the barrel strip detectors the fluences reach $5.3 \times 10^{14} \mathrm{~cm}^{-2}$ for $r=40.7 \mathrm{~cm}$.

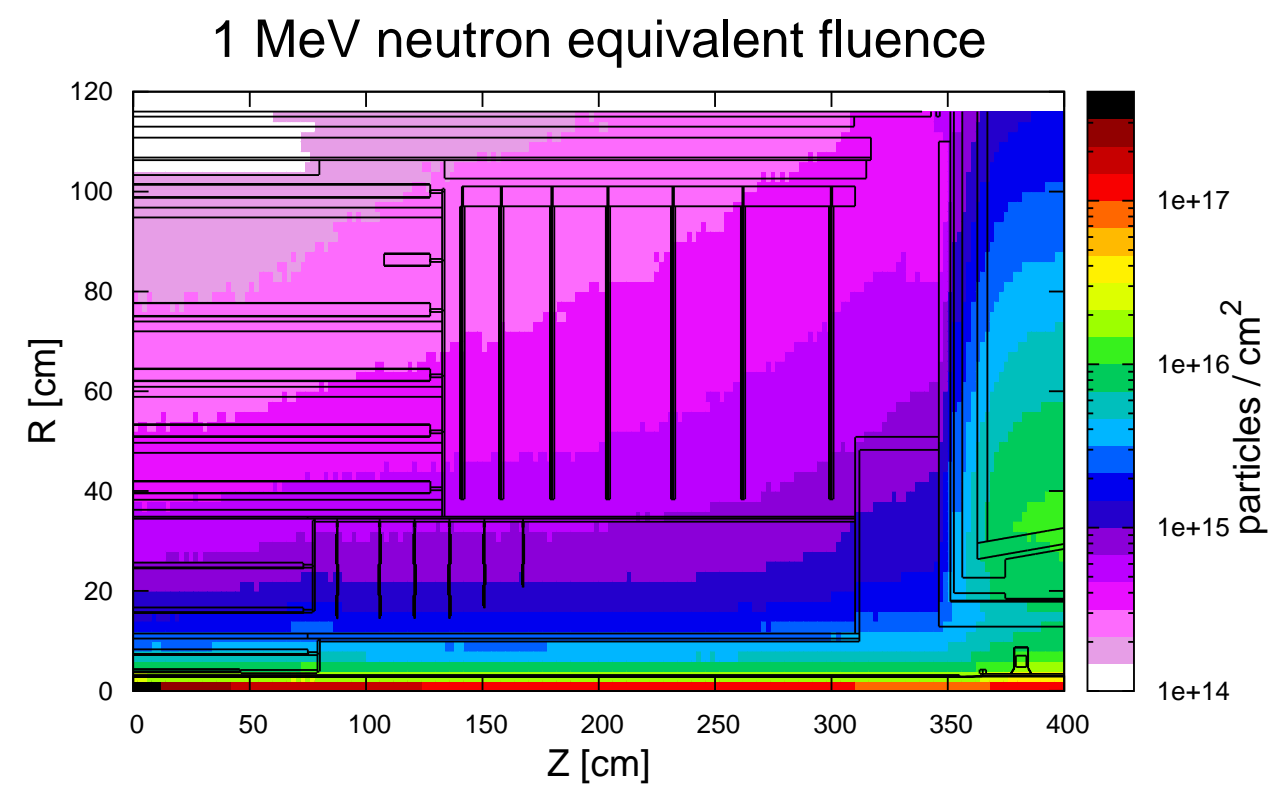

Figure 4: Quarter slice through the inner tracker region of the ATLAS Phase II upgrade geometry constructed in the FLUKA simulations. The colour contours show the $1 \mathrm{MeV}$ neutron equivalent fluences corresponding to an integrated luminosity of $3000 \mathrm{fb}^{-1}$. No safety factors have been applied. 


\subsection{Parameterising the fluences close to the interaction region.}

Close to the interaction point the fluences are dominated by particles coming directly from the collisions, and contributions from secondary interactions in the experiment and machine material is minimal ${ }^{2}$. This has motivated an attempt to parameterise fluences close to the interaction point independent of the hadron collider experiment, based on the predictions of the event generators PYTHIA8 and PHOJET. Furthermore, the event generators predict power law increases in particle production as a function of centre of mass energy, so the parameterisation is extended to cover all collision energies. Fits to the $1 \mathrm{MeV}$ neutron equivalent fluence PYTHIA8 simulations give the following parameterisation as a function of distance $r$ from the beamline:

$$
\Phi_{1 \mathrm{MeV}}\left(\mathrm{cm}^{-2} \mathrm{fb}^{-1}\right)=(\sqrt{s})^{0.37}\left(\frac{1.9 \times 10^{13}}{r^{2}}+\frac{1.6 \times 10^{12}}{r}\right) .
$$

The results for $7 \mathrm{TeV}$ and $14 \mathrm{TeV}$ are shown in shown in Figure 5. Differences between the parameterised fits and simulated data are typically less than 3\%. For particle production constant in pseudorapidity $\eta$, which is approximately the case for the inner tracker coverage, a $1 / r^{2}$ dependence is expected. The impact of the magnet solenoid field is to introduce a $1 / r$ term. It should be stressed that the parameterisation given in Eq. 4.1 was obtained for a $2 \mathrm{~T}$ solenoid field. Further studies are planned to extend the parameterisation to include all values of solenoid fields. The $\sqrt{s}$ power dependence value of 0.37 can be compared to the smaller value of $\sim 0.28$ predicted by PHOJET. The PYTHIA8 parameterisation is preferred because of ongoing improvements to the code for describing inelastic collision data, as well as improved tunings by the LHC experiments.

\section{Conclusions}

High fidelity simulation codes such as FLUKA and GEANT4 are necessary for understanding the complex radiation backgrounds created at hadron collider experiments. Studies have been performed at the new energy regime of the LHC comparing simulation with measurement. Verifying the predictions is important not only for predicting detector performance at the LHC, but also for improving the accuracy of simulations for upgrade studies at the HL-LHC.

The event generators PYTHIA8 and PHOJET-1.12 give similar results when used for radiation background simulations. PYTHIA8 is now the baseline generator of choice due to continued development by the authors to improve the modelling of inelastic proton-proton interactions. The transport codes FLUKA and GEANT4 give similar predictions for the $1 \mathrm{MeV}$ neutron equivalent fluences in the inner detector regions, agreeing typically to $30 \%$, but further study is required to understand differences up to a factor two in the neutron energy spectra.

In general, the level of agreement between simulation and measurement has exceeded expectation. In the ATLAS inner detector regions, differences between fluence and dose predictions and measurements are typically less than $30 \%$. Larger discrepancies up to a factor two (underestimation in predictions) have been observed in some regions and are under investigation. The CMS experiment also reports agreement typically within 30\%, though in the innermost Pixel regions the

\footnotetext{
${ }^{2}$ But if significant material mass is present locally, for example in the Pixel Services, then this assumption may not be valid, due to the regional increase in neutrons produced from hadronic interactions in the material.
} 


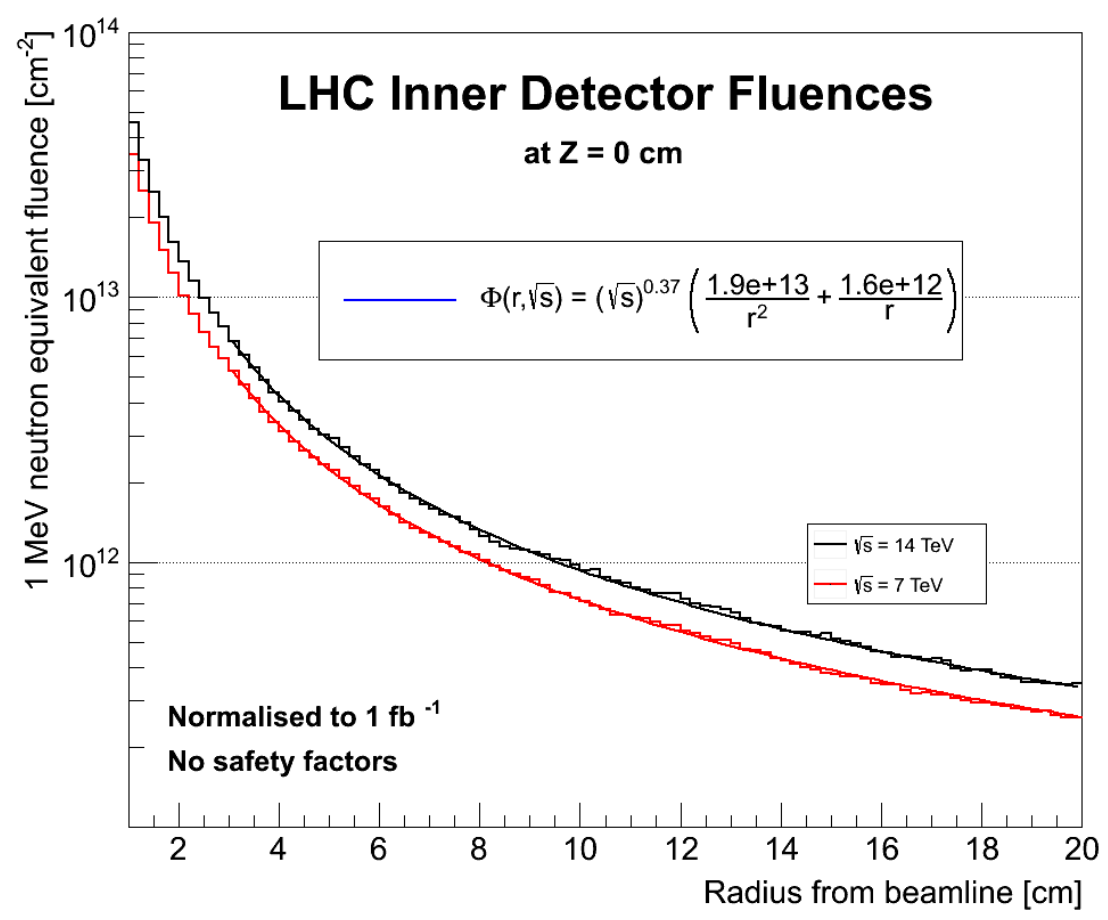

Figure 5: $1 \mathrm{MeV}$ neutron equivalent fluence based on PYTHIA8 simulations and fits for the two centre of mass energies $7 \mathrm{TeV}$ and $14 \mathrm{TeV}$, valid for the central $(\mathrm{Z}=0 \mathrm{~cm})$ barrel regions.

model predictions are 40-70\% higher than the detector measurements [10]. The LHC comparison studies will be repeated for $\sqrt{s}=14 \mathrm{TeV}$.

The radiation backgrounds close to the interaction point of hadron collider experiments are dominated by collision products, and a simple parameterisation of the fluences as a function of radius and collision energy has been demonstrated.

\section{References}

[1] A. Ferrari, P.R. Sala, A. Fasso', and J. Ranft, "FLUKA: a multi-particle transport code", CERN-2005-10 (2005), INFN/TC_05/11, SLAC-R-773; G. Battistoni et al., "The FLUKA code: Description and benchmarking", Proceedings of the Hadronic Shower Simulation Workshop 2006, Fermilab, September 2006, M. Albrow, R. Raja eds., AIP Conference Proceeding 896, 31-49, (2007).

[2] S. Agostinelli et al., "Geant4 - a simulation toolkit", Nucl. Instr. Meth. A 506, 250-303 (2003) ; J. Allison et al., "Geant4 developments and applications", IEEE Transactions on Nuclear Science 53 No. 1, 270-278 (2006).

[3] R. Engel, "Photoproduction within the two component dual parton model: Amplitudes and cross-sections", Z. Phys. C66, 203 (1995); R. Engel and J. Ranft, "Hadronic photon-photon interactions at high energies", Phys. Rev. D54 4244 (1996).

[4] T. Sjostrand, S. Mrenna, and P. Skands, "PYTHIA 6.4 physics and manual", JHEP 05, 026 (2006); T. Sjostrand, S. Mrenna, and P. Skands, "A brief introduction to Pythia 8.1", Comput. Phys. Commun. 178, 852-867 (2008), arXiv:0710.3820 [hep-ph]. 
[5] The ATLAS Collaboration, "The ATLAS Experiment at the CERN Large Hadron Collider", JINST 3, S08003 (2008).

[6] The ATLAS Collaboration, "Measurement of the inelastic proton-proton cross-section at $\sqrt{s}=7 \mathrm{TeV}$ with the ATLAS detector", Nature Communications 2, 463, (2011).

[7] M. Bosman, I. Dawson, V. Hedberg, M Shupe, "ATLAS Radiation Background Taskforce Final Summary Document", ATL-GEN-2005-001 (2005).

[8] R. Harper et al., "Evolution of silicon microstrip detector currents during proton irradiation at the CERN PS", Nucl. Instr. Meth. A 479, 548 (2002).

[9] M. Moll et al., "Leakage current of hadron irradiated silicon detectors - material dependence", Nucl. Instr. Meth. A 426, 87 (1999).

[10] S. Gibson, "Radiation Damage to Currently Running LHC Silicon Detectors", these proceedings.

[11] I. Mandic et al., "First Results from the Online Radiation Dose Monitoring System in ATLAS experiment", IEEE Nuclear Science Symposium and Medical Imaging Conference, Conference Record, NP3.M-40 (2011).

[12] J. Bernabeu, "ATLAS strip upgrade", these proceedings. 